\title{
An Investigation into the Next Generation of High Density, Ultra High Voltage, Power Supplies
}

\author{
R. E. P. Frost, and P. L. Lewin \\ Electronics and Computer Science \\ University of Southampton \\ Southampton, United Kingdom \\ Rf6g13@soton.ac.uk
}

\author{
M. Spong \\ QinetiQ \\ Cody Technology Park \\ Farnborough, United Kingdom
}

\begin{abstract}
This paper details an investigation into possible technologies suitable for a DC power supply that is comparable in size and voltage output to a Cockcroft Walton Generator, but can produce much greater currents and stores only a fraction of the energy. Early work suggested that the most suitable technologies were those based on conventional transformers, specifically Insulated Core Transformers (ICTs) and High Frequency Cascade Transformers (HFCTs); both of which were investigated further.
\end{abstract}

Different methods have been used to calculate the flux loss in an ICT and ways of reducing these losses investigated. It is concluded that the flux loss of an ICT is too great for use in a practical, high power density, power supply, and that future work in this field should focus on an HFCT based design.

Keywords - Insulated Core Transformers, Flux Loss, Cascade Transformers, Circuit Equivalent Model

\section{INTRODUCTION}

There is growing interest, from both industry and academia, for a DC power supply capable of outputting $2 \mathrm{MW}$ at $1 \mathrm{MV}$. Ideally, the geographic footprint of such a power supply would be as small as possible, meaning that the design will need a high power density. If a fault, such as a short circuit, were to occur in a system that stored a lot of energy the results could be catastrophic. For this reason, there is a design requirement that the amount of stored energy within the supply should be kept to a minimum.

Technologies, such as Cockcroft Walton Generators, Nested High Voltage Generators, and kinetic energy based systems were considered, but dismissed as they either suffered from heavy voltage drop under high load, stored too much energy, or both. Of the designs considered, those based on conventional transformers, specifically Insulated Core Transformers (ICTs) [1] and High Frequency Cascade Transformers (HFCTs) [2], showed the most promise and were, therefore selected for further investigation.

The size and voltage ripple of these technologies can be reduced by including them in designs that use power electronics to increase the operating frequency into the region of kilohertz, rather than the tens of hertz grid frequency. Voltage ripple, and therefore the strain on the rectification circuit, can be further reduced by connecting these transformers in parallel and operating them out of phase with each other.

An additional design constraint that was placed on this project was that the power supply should be supplied from a $415 \mathrm{~V}$ three phase mains outlet. This means that the overall turns ratio in each transformer must be 1:2,500. For initial comparison, it was decided that the primary side should consist of 4 turns, while the secondary circuit would be made up of 100 separate windings, each consisting of 100 turns, printed on PCBs.

\section{OPTIMISING ICT LAYOUT}

In theory, a transformer could be designed that has a single low voltage primary winding and numerous secondary windings. These secondary windings could then be connected in series in order to produce any desired output voltage. However, in reality additional secondary windings place an additional strain on the insulation, as the potential increases between the $\mathrm{HV}$ windings and the grounded core. It has been suggested that the practical limit to the voltage between the HV winding and the core is $200 \mathrm{kV}$ [3].

One way to solve this problem is to put electrically insulated gaps in the core, so that sections can be held at different potentials, thus reducing the strain on the insulation. This reduction in strain on the insulation does, however, come at the cost of the permeability of the flux path as the insulated gaps have a lower magnetic permeability than the core. This reduction in permeability leads to greater leakage flux in the core, meaning that secondary windings located further away from the primary winding will produce a lower voltage per winding section as a result of reductions in core magnetic flux.

Different techniques have been used to reduce the voltage drop caused by flux loss in ICTs. Van de Graaf used more turns in secondary windings located further up the stack from the primary [1], however this has the drawback of adding complexity to the system design as each secondary winding must be designed specifically for its position in the stack. Cross added capacitors into the secondary winding circuits, that would store energy to compensate for the flux lost in the system [3]; this would be unsuitable for a high power application, in which stored energy must be kept to a 
minimum. The work reported in this paper focuses on methodologies for minimising flux leakage in a system [4].

Four different core designs were investigated to see which was the most effective at reducing flux loss. Each design was drawn in two dimensions in the FEA software package, MagNet, and simulated under static conditions. Ten windings were used, each consisting of 100 turns, in each simulation, with the bottom winding being used as a primary winding. The windings are each wrapped around an insulated section of ferrous core, which is $80 \mathrm{~mm}$ across and $9 \mathrm{~mm}$ thick, and is insulated from its surrounding cores by a $1 \mathrm{~mm}$ thick gap.

The first layout to be considered is the basic stack, as shown in Fig. 1. The second is a variation of this layout that incorporates flux diverters [5]. Flux diverters are coils of wire that are wrapped around the gaps between cores. A cross section of such devices is shown in Fig. 2. When an alternating magnetic field passes through these coils, it induces an electrical current in the coil. This current, in turn, induces a magnetic field around it that resists the flow of flux. In this way, the coils channel the magnetic flux through their centre. As the FEA simulations are being conducted in a static time frame, and no current is being induced in the coil, for the purposes of the simulation, a DC current, representing the peak AC value, was set to act on each flux diverter.

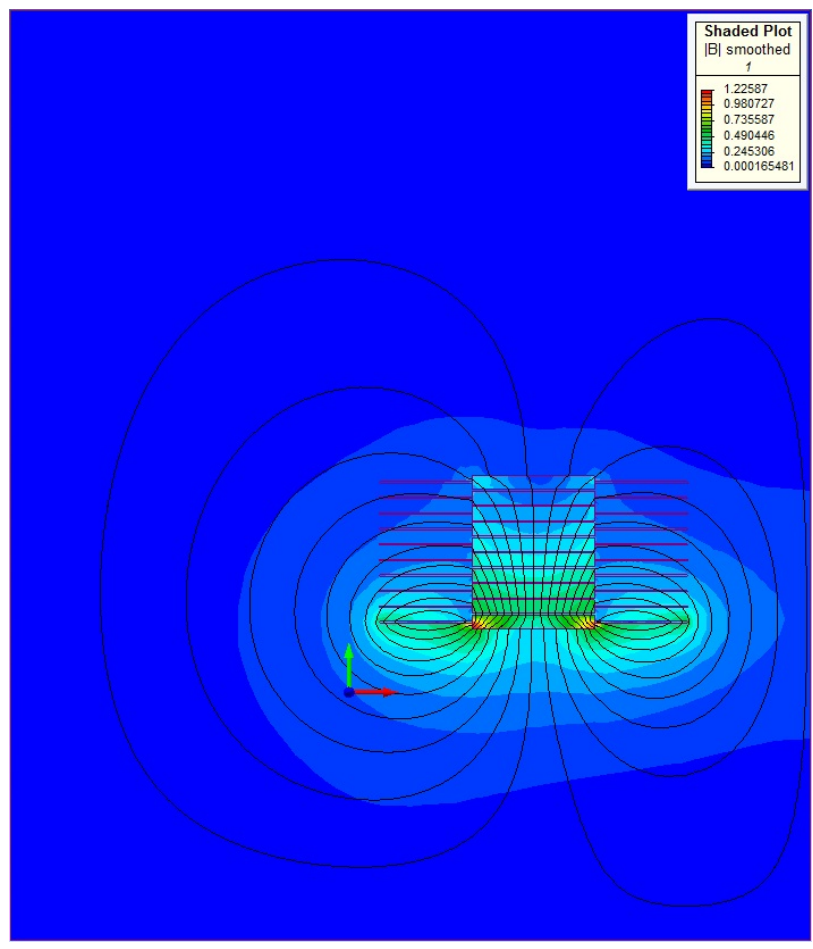

Fig. 1. The flux flow around a 10 layer ICT

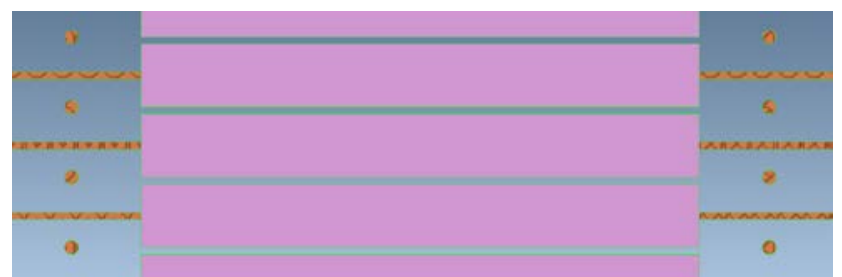

Fig. 2. Flux diverters surrounding a stack of insulated cores
In the designs based around conventional stacks the bottom section of core is grounded, while the potential in each subsequent section increases until the top core is maintained at the maximum potential of the design. If there is no ideal flux return path, as in shell and E-Type transformer cores, the flux will be ill inclined to pass through the air surrounding the primary winding, as seen in Fig. 1. One possible solution to this is to construct a second, inverted, core stack above the main stack. The top and bottom cores of this new stack can then be grounded, while the centremost core can reach the highest voltage. This combined stack can then be driven from a primary winding driven around both the top and the bottom. Flux can then pass between the two primary windings through the core stack, to produce a more consistent flux density through each secondary winding. This design, and subsequent flux flow, is shown in Fig. 3.

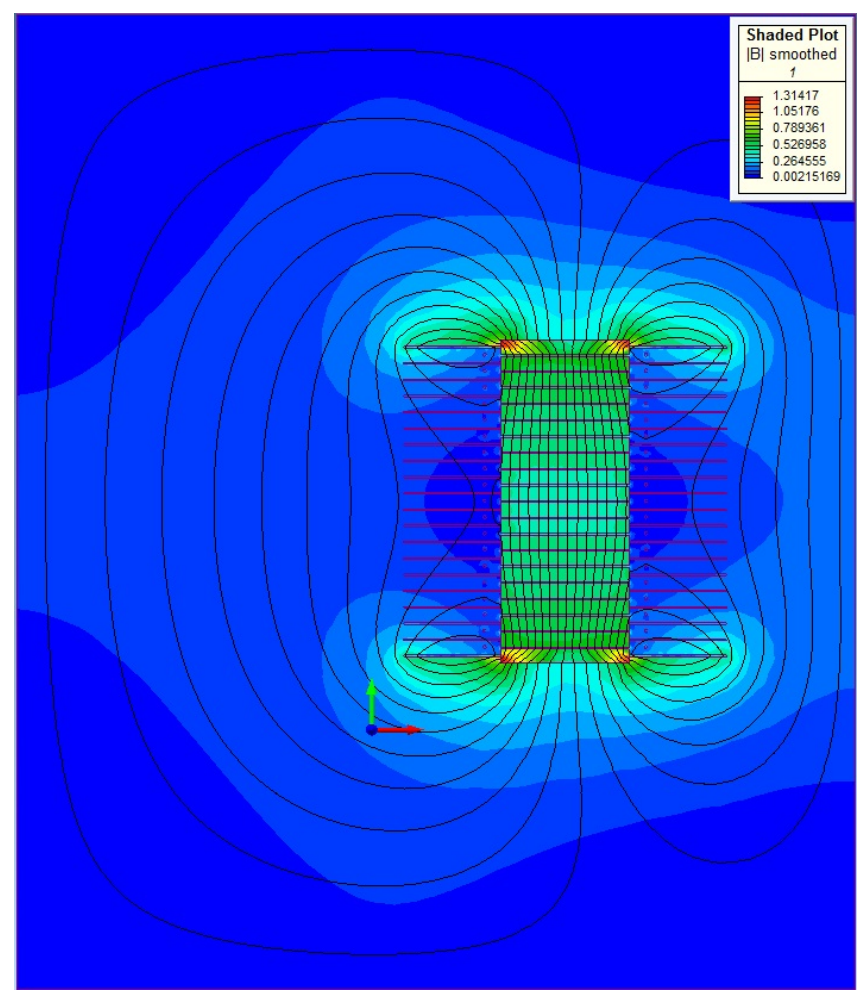

Fig. 3. The flux flow around a 20 layer ICT with flux diverters, and primary windings at the top and bottom.

An improvement on this design would be to place a flux return path, constructed from a material with a high relative permeability, around the stack to form a complete, closed flux path through the windings. This layout, as well as the resultant magnetic field, can be seen in Fig. 4.

The greatest drawback with using this clamped return path, is the amount of material needed for its construction. A way of reducing the amount of material needed would be to construct a partial flux return clamp, consisting of the two yokes, but omitting the second limb of the full clamp; this reduces the amount of material required. This design, as well as the flux flow, can be seen in Fig. 5.

The minimum normalised flux linkage formed in each of these designs is shown in Fig. 6, for both a single and double 
primary winding configuration. From this it can be seen that having a second primary winding has the greatest affect on reducing flux loss. The design with the lowest flux loss is the double primary, clamped return path. This will be discussed further in later sections.

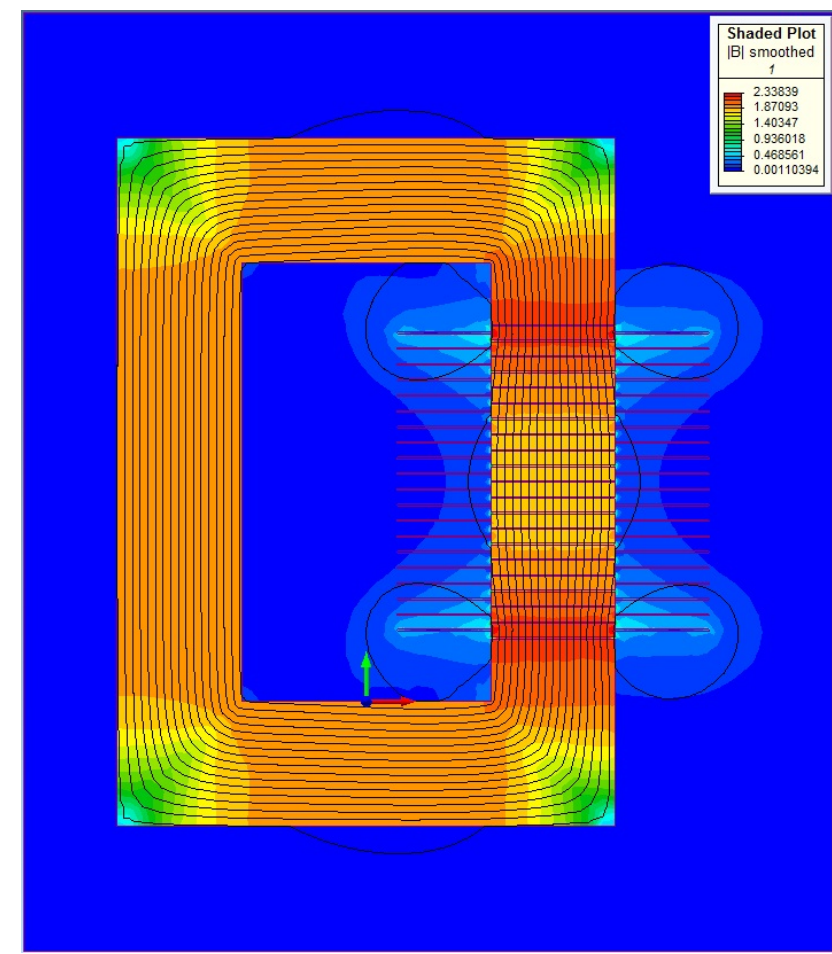

Fig. 4. The flux flow around a 20 layer ICT with a flux return clamp, and primary windings at the top and bottom.

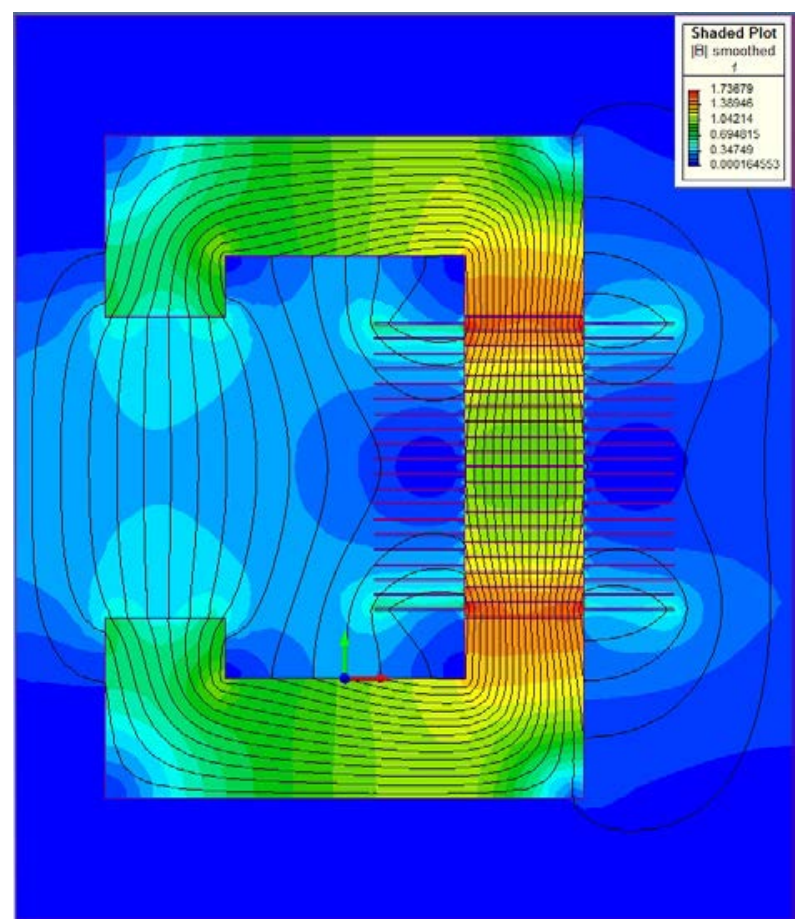

Fig. 5. The flux flow around a 20 layer ICT with a partial flux return clamp, and primary windings at the top and bottom.

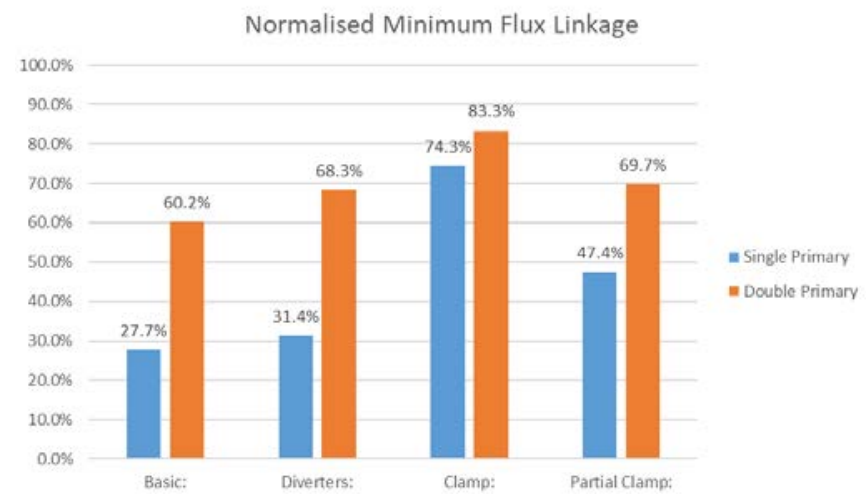

Fig. 6. Minimum flux linkage found in each ICT as a percentage of the primary winding flux linkage.

\section{MethodS FOR CALCUlATING FluX LOSS}

In order to assess their suitability for HV applications, a series of ICTs were simulated with stack heights ranging from 1 to 100 . Two different methods were used to predict the flux loss in these transformers. The first of these was an analytical technique based on magnetic flux equivalent circuits. The second was FEA performed in MagNet. The results of these two methods were then compared in order to assess their validity.

\section{A. Flux Circuit Model}

Though FEA is known to produce accurate results, it is slow and computationally intense; this means that, although it is ideal for confirming results, it is not suitable for the design stage of a large system. For this a technique is required that can give quick and approximate predictions of flux loss for a system with multiple variables, using only simple calculations.

A flux equivalent circuit [6] works by creating an equivalency between the flow of magnetic flux in a transformer system and an electrical circuit. In this way, magnetic reluctances can be converted into electrical resistances, $R_{\mu}$, using

$$
R_{\mu}=l \cdot(\mu S)^{-1}
$$

where $l$ is the length of the flux path through a material; $S$ is the cross sectional area of the material; and $\mu$ is the materials permeability.

The flux is assumed to move around the clamped return path ICT as shown in Fig. 7, with the majority of the flux passing directly up the stack, and leakage flux passing straight between the insulated core sections and the return path. As the permeability of the core material is much greater than that of the insulation surrounding them, the core sections can be modelled as perfect electrical conductors, while the air gaps are equivalent to resistances. In this way a clamped return path ICT can be modelled as a ladder circuit, as shown in Fig. 8 with the flux return clamp acting as a ground plane, the core sections acting as nodes and the insulation gaps acting as resistors.

Once the magnetic equivalent circuit has been constructed, the magnetic field strength can be calculated in much the same 
way as current in an electrical circuit. At this point, the key concern is with the percentage of flux that is lost in a design, consequently all results are normalised and so it is not necessary to calculate the precise magnetic field strength in the circuit.

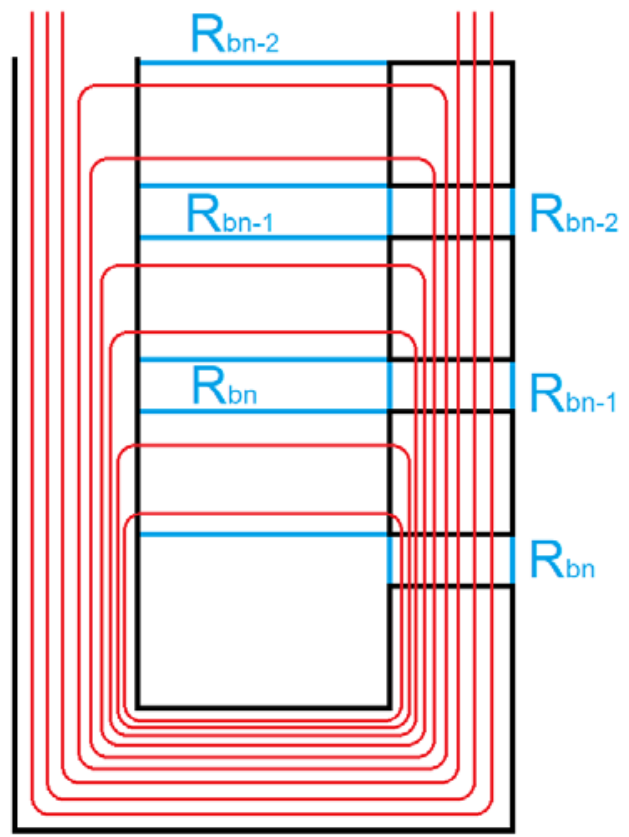

Fig. 7. The flow of flux in a clamped return path ICT, as modelled by the Flux Circuit Model.

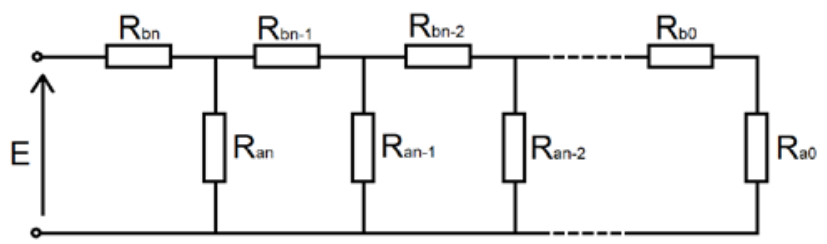

Fig. 8. The flux circuit equivalent model of a clamped return path ICT.

\section{B. FEA Simulation}

FEA simulations were performed using Infolitica MagNet, using static 2D simulation. A macro was written that would construct clamped return path ICTs of varying heights, between 1 and 100 stages, simulate them and record the results. It is worth noting that when drawing clamped return path ICTs, twice as many cores need to be drawn as with the flux equivalency circuit. This is because the core stack needs to be reflected in order for it to be grounded at both ends.

\section{Comparison of Techniques}

A series of clamped return path ICTs were simulated, using both the magnetic flux circuit and FEA. The insulated core sections had a cross section of $80 \mathrm{~mm}$ by $80 \mathrm{~mm}$, with a $1 \mathrm{~mm}$ insulation gap between each one. There was a gap of $160 \mathrm{~mm}$ between the insulated core stack and the flux return clamp. An example of an ICT with 100 stages, that meet these dimensions is shown in Fig. 9. A comparison of the percentage flux losses, found using both methods, is shown in Fig. 10.

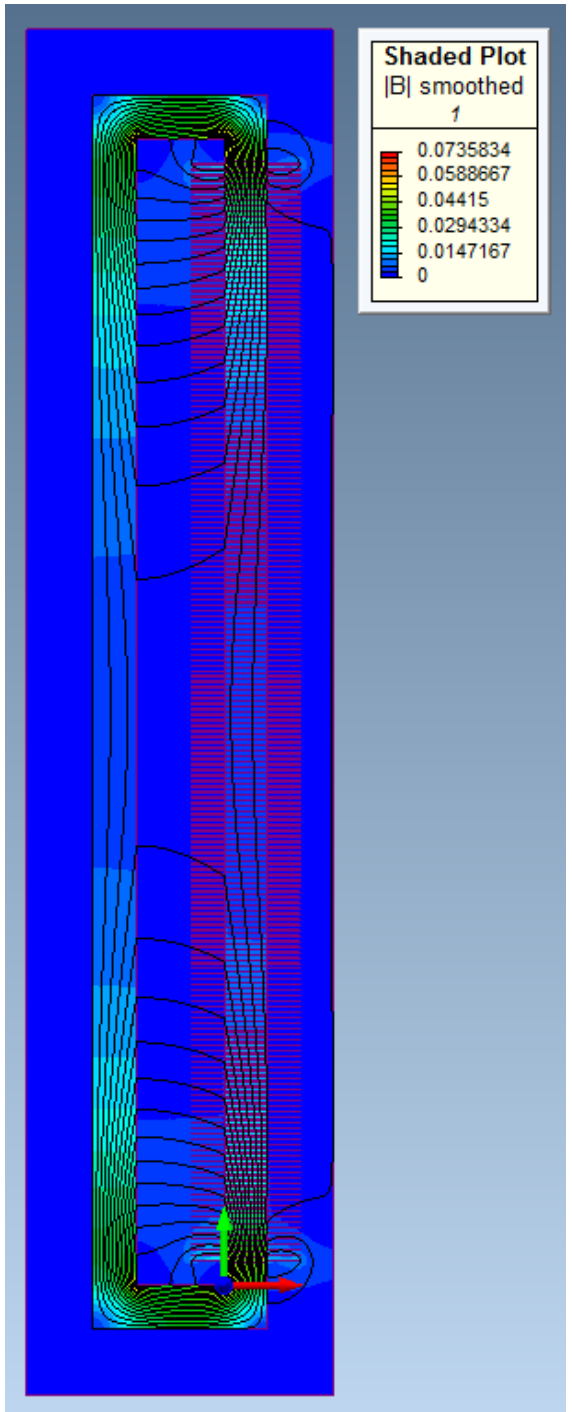

Fig. 9. A 100 stage, clamped return path ICT and the flux flow around it.

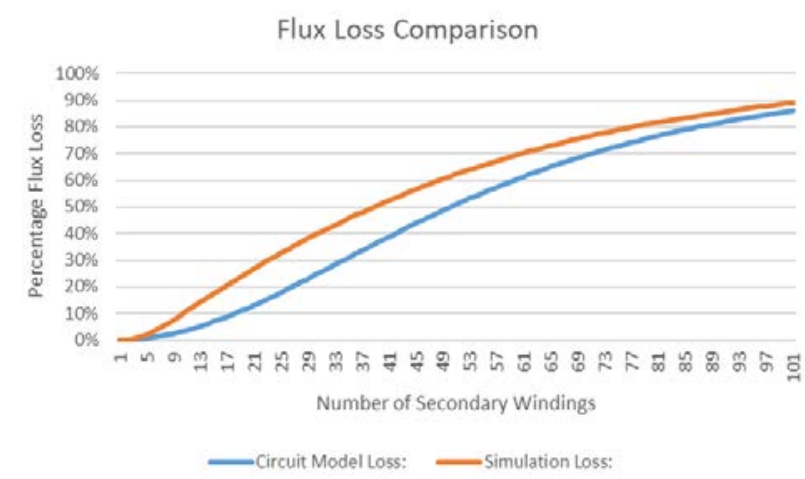

Fig. 10. The maximum loss of flux linkage in clamped return path ICTs with an incrementally increasing number of secondary windings, predicted using both the flux circuit model and FEA.

From Fig. 10 it can be seen that both methods have a strong correlation, with there only being 3\% difference between the two methods for a stack of 100 cores. It is worth noting that 
there is a greater difference between the results when there are fewer cores. This is because the magnetic circuit model does not take into account the leakage flux that passes from the cores on the end of the stack, to the horizontal yokes of the flux return clamp, rather than flowing all the way to the vertical limb. As the stack grows in height, the cores towards the centre of the stack are closer to the limb than the yokes of the flux return clamp and therefore the flux does indeed pass straight to the limb. The flux that passes to the yokes therefore account for a smaller proportion of the overall flux loss.

Unfortunately, both techniques predict unworkably large flux losses for a practical power supply. The conclusion is that ICTs are therefore unsuitable without some form of additional design variation.

\section{FURTHER CHANGES TO ICT LAYOUT}

It isn't necessary for each secondary winding to be wrapped around an insulated core of its own. Flux loss might be reduced by grouping two or more secondary windings around each segment of insulated core to form a stage. This would reduce the number of necessary insulated cores, but require additional insulation between each stage as there will now be a greater potential acting on each section of insulated core. As well as this, the reluctance between each section of core and the flux return clamp will be reduced as each section will have to have a greater surface area to accommodate the additional windings. This leads to greater flux loss between each stage.

The flux circuit equivalent model of a return clamped ICT consists of a resistor ladder. Increasing the stage sizes translates to lumping the circuit parameters together, creating a circuit with an equivalent resistance. As any ladder circuit that models a flux return clamped ICT will have the same circuit parameters, they will therefore predict the same flux loss. The flux circuit model can, therefore, only model flux return clamped ICTs based on their number of windings, cross sectional area of the insulated cores, thickness of core and insulation per winding, and distance between the core stack and flux return clamp. The number of windings sharing a core has little effect on the flux loss predicted by the flux circuit model. This is in contrast to FEA analysis, as seen in Fig 11, which sees a reduction in flux loss as the stage size increases. However, the reduction in flux loss from an ICT with a stage size of 1 , to a stage size of 20 , is only from $89 \%$ to $81 \%$. Both of these values are unworkably high.

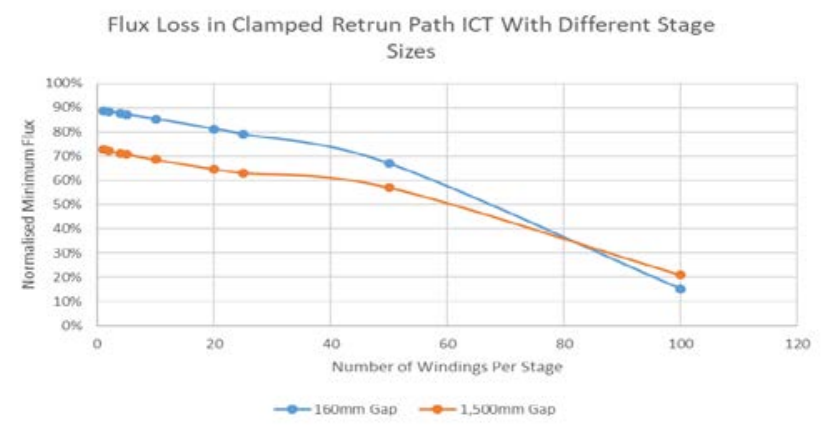

Fig. 11. The maximum loss of flux linkage in clamped return patth ICTs with different stage sizes and different gaps between the core stacks and the flux retun clamp limb, calculated using FEA.
The single biggest source of flux loss in a clamped return path ICT is the relatively narrow gap between the insulated core stack and the flux return path. This source of loss has been advantageous in its predictability, allowing the magnetic circuit model to be so accurate, but is unacceptable due to the magnitude of the losses it causes. In order to reduce losses further, the gap between the stack and the return clamp, was widened from $160 \mathrm{~mm}$ to $1,500 \mathrm{~mm}$. As the gap between the stack and return clamp widens, the flux path becomes less predictable as more flux passes from the core stack to the yokes of the flux return clamp, instead of the limb, meaning that the flux circuit model becomes less accurate. In this case, with a stage size of 20, the flux circuit model predicted peak flux loss of $33 \%$ while FEA, results of which can be seen in Fig. 11, predicted a loss of $65 \%$.

Even using stage sizes of 20, and with a 1,500mm gap between the core stack and the flux return clamp, the flux loss is still unacceptably high. Additionally, the discrepancy between the results found using the two methods highlights that the flux circuit model is only an effective tool when the flux path in a system is predictable.

\section{LiMitATION OF MODELS}

Both models were limited by being implemented in only two dimensions. Therefore, they only take into account flux that is lost in a flat plane. In reality, flux will fan out between the core stack and the flux return clamp, effectively increasing the cross sectional area of the insulated gap between the two and thus reducing the reluctance. Another limitation, which was accounted for in FEA but not in the flux circuit equivalent model, was flux that would return to the primary winding on the opposite side of the core stack to the flux return clamp.

Both of these improvements have been omitted from this study as they would increase the calculated flux loss. As the flux loss has already been deemed to be unacceptably high, using the most optimistic models, development of a more pessimistic one would not significantly affect the conclusions drawn from this study.

\section{High FREQUENCY CASCADE TRANSFORMERS}

Unlike an ICT, which passes flux to the next core through an insulated gap, cascade transformers use windings that are assumed to be lossless. For this reason, there is no equivalent to the flux circuit model that can be applied to cascade transformers. FEA ssimulation and practical experiments are the only way of calculating flux loss.

A cascade transformer, consisting of 100 secondary windings split in to five stages, is shown in Fig. 12. For simplicity, the secondary windings seen on either side of each core represent ten (100 turn) windings. The auxiliary winding, on the top of each core, connects to the primary winding on the bottom of the core above. The direction of windings changes between each successive transformer, ensuring that the flux changes direction between each core. This is important as it ensures that the flux in parallel yokes is flowing in the same direction, minimising the flux leakage between the two cores. As transformer windings only work in an alternating magnetic 
field, Time-Harmonic 2D simulation was, therefore, used to predict the flux loss found in each stage of the cascade transformer. The results are listed in table 1.

TABLE I FLUX LOSS IN HFCT

\begin{tabular}{|c|c|c|}
\hline Core: & Flux Linkage (Wb): & Percentage Drop (\%): \\
\hline 1 & 13.27 & 0 \\
\hline 2 & 12.84 & 3.23 \\
\hline 3 & 12.53 & 5.56 \\
\hline 4 & 12.33 & 7.07 \\
\hline 5 & 12.23 & 7.81 \\
\hline
\end{tabular}

It can be seen that the peak flux loss can be reduced to $7.81 \%$, using a cascade transformer, compared with 33\% using the most optimistic simulation of an ICT design.

\section{CONCLUSIONS}

It can be concluded that ICTs are not suitable for use in a $1 \mathrm{MV}, 2 \mathrm{~A}$ power supply as the excessive flux loss, causes an insurmountable voltage drop in the windings located furthest away from the primary winding. Far more promise was shown by HFCTs, which have many advantages over ICTs. Most importantly that the closed magnetic paths significantly reduce the flux loss and therefore voltage drop.

In addition to this, it was found that the flux circuit model is a useful tool in designing clamped return path ICTs, by reducing the number of FEA simulations that need to be performed, providing the flow of leakage flux is known to be predictable.

\section{VIII.REFERENCES}

[1] R. J. Van de Graaff, "High Voltage Electromagnetic Apparatus Having an Insulating Magnetic Core,” 3289066, 1952.

[2] B. Y. R. W. Sorensen, "Development and Characteristics of a 1,000,000-,” J. A.I.E.E, vol. 44, no. 4, pp. 373-378, 1925.

[3] J. D. Cross, "Modular High Voltage Power Supply with Integral Flux Leakage Compensation,” US6026004A, 1998.

[4] D. J. Swaffield, P. L. Lewin, G. Chen, and J. K. Sykulski, "Cryogenic Dielectrics and HTS Power Aparatus: Research at the Univerity of Southampton,” IEEE Electr. Insul. Mag., vol. 22, no. 5, pp. 29-37, 2006.

[5] L. D. Barry, "Flux Diverter,” US3072750, 1953.

[6] P. Hammond and J. K. Sykulski, Engineering Electromagnetism Physical Processes and Computation. Oxford: Oxford University Press, 1994.

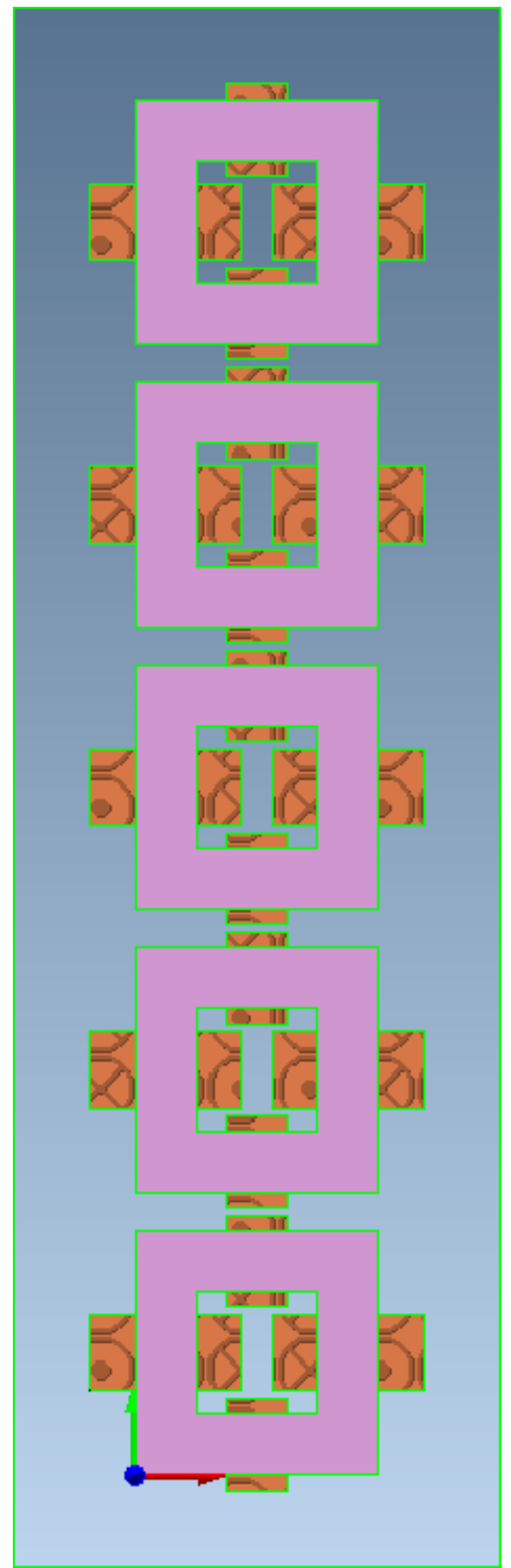

Fig. 12. A model of a five stage cascade transformer. 